\title{
EVALUACIÓN QUÍMICA Y MICROBIOLÓGICA DE LA PLAYA DE “LOS ESTEROS" EN LA CIUDAD DE MANTA
}

\author{
Chemical AND microbiological EVAluation of THE “Los Esteros" \\ BEACH, IN THE MANTA CITY
}

Carlos Enrique González Arteaga ${ }^{1, *}$ e Hilda Yajaira González Arteaga ${ }^{2}$

\author{
${ }^{1}$ Pontificia Universidad Católica del Ecuador, Campus Chone. Dirección, Vía a Quito Km 111/2, Teléfono: (593) 099 482-280. Chone, \\ Ecuador. \\ ${ }^{2}$ Facultad de Medicina de la Universidad Laica Eloy Alfaro de Manabí. Dirección Av. Circunvalación, Manta, Ecuador. \\ *Autor para correspondencia: krloseng@hotmail.com
}

Manuscrito recibido el 20 de enero de 2015. Aceptado, tras revisión, el 10 de junio de 2016

\begin{abstract}
Resumen
Esta investigación analiza el estado ambiental de las playas de la parroquia "Los Esteros" de la ciudad de Manta. Éstas playas en sus $1.2 \mathrm{~km}$ de extensión aproximada, presentan la cantidad de $\mathrm{DBO}_{5}, \mathrm{DQO}$, sólidos suspendidos así como coliformes totales, Escherichia coli entre otros factores. Los cuales inciden en las características de las aguas y sean consideradas poco aptas para usos recreativos de contacto primario. El análisis se lleva a cabo en el año 2014, utilizando las normas establecidas por el índice de calidad del agua y la comparación con los límites máximos permisibles para descargas a cuerpos marinos, establecidos en la Ley de Gestión Ambiental, del Ecuador.
\end{abstract}

Palabras claves: Coliformes totales, Límites máximos permisibles, playa Los Esteros, Manta.

\begin{abstract}
This research analyzes the environmental state of the "Los Esteros" beach in the city of Manta. In this beach, with $1.2 \mathrm{~km}$ length, were sudied the amounts of $\mathrm{DBO}_{5}, \mathrm{DQO}$, suspended solids and total coliforms; included Escherichia coli and other factors that affect the characteristics of the water and can considered unsuited for primary contact and recreation. The analysis was carried out in 2014, using the standards set by the water quality index and maximum permissible limits for discharges to marine bodies established by the Environmental Management Law of Ecuador.
\end{abstract}

Keywords: Total Coliforms, Maximum permissible limits, Los Esteros beach, Manta.

Forma sugerida de citar: González, C. y H. González. 2016. Evaluación química y microbiológica de la playa de “Los Esteros" en la ciudad de Manta. La Granja: Revista de Ciencias de la Vida. Vol. 23(1): 47-52. ISSN impreso: 1390-3799. ISSN electrónico: 1390-8596. 


\section{Introducción}

En la parroquia "Los Esteros" el mayor problema son las descargas de aguas residuales, emisiones de humo y gas tóxico producido por actividades humanas e industriales aledañas que cada vez aumentan y con ello contribuyen a una mayor degradación de estas zonas no solo turísticas sino también ecológicas.

Además, existen bypass sanitarios, como por ejemplo el construido a la altura de la Calle 119 y Av. 101 (Ver Figura 1) y que lleva a que las aguas negras vayan directamente al mar, y otros bypass que se han construido en diferentes lugares a lo largo de esta playa y no solo afectan a la misma sino que se unen con los agentes contaminantes generados de la playa de Tarqui.

Es importante poder determinar el tipo de contaminación que se tiene sobre el área de estudio para tomar las medidas correctivas y preventivas, y aplicar la remediación pertinente, la misma que si bien es cierto será complicada, pero con resultados notorios. Con es- tas medidas se debería también iniciar al mismo tiempo una cadena progresiva de campañas agresivas de concientización ambiental.

De esta manera, es evidente la importancia de realizar procedimientos de análisis cualitativos y cuantitativos que permitan determinar el tipo y la concentración del contaminante; además, de su fuente de generación. Para después dar respuesta al pregunta de por qué en los últimos años, según reportes del subcentro de salud de la parroquia Los Esteros, han surgido varias enfermedades dérmicas e intestinales en la población aledaña a esta playa sobre todo en la infantil (González, 2014).

La contaminación de la playa de la parroquia Los Esteros de la ciudad de Manta, es un problema que viene afectando tanto a la fauna marina como a los habitantes, turistas del sector y a los legendarios pescadores que iniciaron esta parroquia hace algunas décadas atrás. La situación empeora en la playa ubicada en la desembocadura del Río Muerto ya que es el medio que utilizan los industriales de la parroquia y

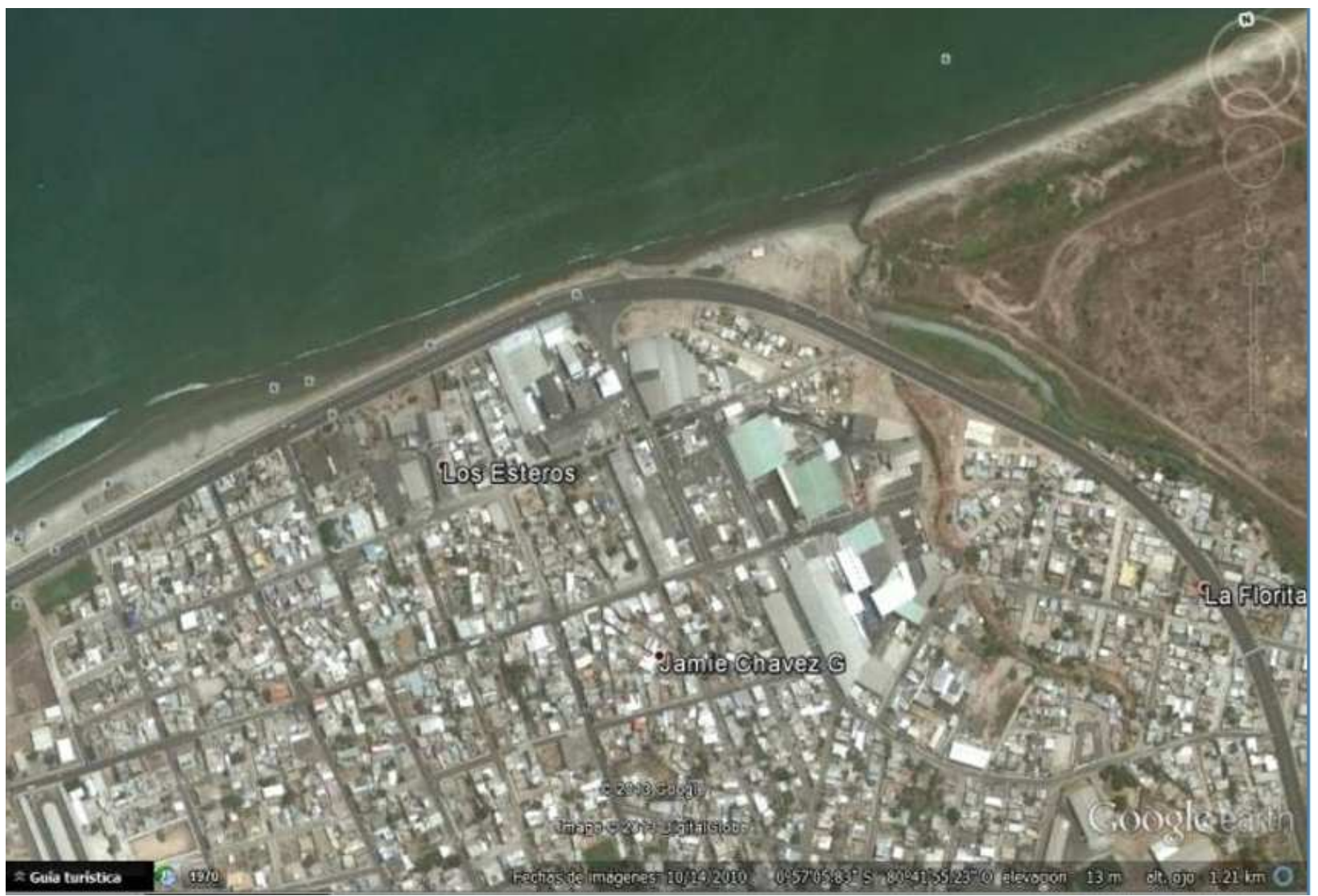

Figura 1. Playa “Los Esteros". Fuente: Google maps (2010) 
algunos habitantes para descargar aguas residuales y que tienen destino final el mar. Inclusive la gente de la zona indica vernáculamente que es "el río Muerto que mató". Según las investigaciones realizadas por los autores de este artículo y por el Biólogo Jorge González (2014), esta playa registra los más altos índices y niveles de contaminación permitidos por la Ley de Gestión Ambiental. Puesto que existen altas dosis de grasas, aceites, enterobacterias, E. coli y hasta coliformes fecales. Pese a que los niveles han disminuido en los últimos años, aún hay conexiones clandestinas de fábricas y domicilios que van directo al río.

Las aguas no deben registrar niveles de contaminantes por encima de lo permitido, esto es en DBO100 $\mathrm{mg} / \mathrm{lt}$, y DQO $250 \mathrm{mg} / \mathrm{lt}$ así como 100 coliformes fecales por cada $100 \mathrm{mg} / \mathrm{lt}$ de sólidos suspendidos según la ley para la Prevención y Control de la Contaminación Ambiental del Ecuador (2008), al superar estas cantidades de residuos se convierten en aguas no aptas para la natación.

En el caso de esta playa como en muchos cuerpos de agua marino en el mundo, los vertidos llegan directamente al mar de la misma manera como salen de sus fuentes de origen, además de los contaminantes que se le suman en el trayecto que realizan (Daiefa et al., November 2014).

El agua de los mares y de los ríos ha sido utilizada tradicionalmente, como medio de evacuación de los desperdicios humanos y los ciclos biológicos del agua aseguran la reabsorción de dichos residuos orgánicos reciclables. Algunos de los productos vertidos, no son biodegradables y permanecen largo tiempo en el ambiente. Los elementos vivos que habitan en estas aguas, usualmente ingieren estos microorganismos o compuestos químicos y los llevan en la cadena trófica alimentaria de nuevo al ser humano. Específicamente en esta playa, se realizan labores de pesca y en el mismo sitio se comercializa pescado supuestamente fresco generando efectos negativos para la salud humana, aparte del daño ecológico en general. Efectos negativos que incluso ya se reflejan según el ministerio de salud a través del subcentro de salud de la parroquia donde se encuentra ubicada la playa en mención (González, 2014).

Hidrográficamente, el sitio de estudio se encuentra atravesado por tres extensos ríos invernales (Manta, Burro y Muerto), los que cruzan amplias zonas urbanas pobladas, y desembocan en el mar generando malos olores, color del agua con alteraciones, incrementándose los valores permitidos por la (Ministerio del Ambiente del Ecuador (MAE), 2008) en cuanto a DBO y DQO, variaciones en el $\mathrm{pH}$, entre otros. El mayor foco de contaminación de esta playa lo constituye el ya mencionado cauce del Río Muerto. Este río, por ser invernal, la mayor parte del año pasa seco, al menos así debería ser, incluso por eso su nombre, además de por la poca cantidad de vida marina que se encuentra en él. Sin embargo, al cruzar por zonas pobladas tales como "Mar y Cielo", "Divino Niño", "Urbanización Millenium" y la "Florita", los cauces del río han sido tomados como canales de descargas de aguas servidas, ya que una buena parte de la población circundante, ha construido canales o tuberías directas al cauce del río y de esta manera evacuan sus aguas negras y desechos sólidos, aun sabiendo que estos contaminan y los afectan directamente a ellos mismos.

Una parte de estas aguas servidas, al no existir flujo alguno de lluvias durante el verano, que las arrastren consigo hacia el mar como en el sector de las tres cruces, permanecen estancadas largos lapsos de tiempo, emanando fuertes olores y contaminando el ambiente, lo que lo convierte en un gran caldo de cultivo de insectos, roedores y agentes causantes de enfermedades que generalmente ponen en riesgo la salud de los mismos moradores del sector.

Así, la falta de una educación ambiental comunitaria en Salud Pública y el desconocimiento de los peligros que aquello representa para la propia salud de las personas que habitan en estos sectores, impide que se tome conciencia de las nefastas consecuencias y de las malas costumbres ciudadanas, lo cual se convierte en un verdadero peligro. A pesar de que se encuentra en construcción el plan maestro hidrosanitario y el mismo que casualmente cubre la mayoría de barrios que son atravesados por el río Muerto y cercanos a la playa en estudio (Empresa Pública de Aguas de Manta, 05 de 11 de 2014).

\section{Materiales y métodos}

El presente trabajo investigativo se realizó en los 1.2 $\mathrm{km}$ de playa que comprende el sector de la parroquia "Los Esteros" del cantón Manta. Los análisis realizados comprenden: Composición del agua de la playa, bromatología, parámetros microbiológicos, $\mathrm{pH}$ y temperatura.

Cada muestreo se realizó en tres puntos, El punto de Muestreo 1 se ubicó en la desembocadura del río Muerto, que es el lugar donde se une con la playa de los Esteros, el punto de Muestreo 2, ubicó en la zona de mayor afluencia turística, y el punto de Muestreo 3 
se ubicó al final de la playa de dicha parroquia y donde inicia la playa de la parroquia Tarqui.

En cada lugar de muestreo se realizaron tres repeticiones con un intervalo de 10 a 30 días correspondientes a los meses de octubre, noviembre y diciembre de 2014. Meses en los que se presenta un cambio de temporada de verano a invierno.Durante este periodo se realizó una medición diaria de $\mathrm{pH}$, temperatura, sólidos totales, DBO, DQO, E. Coli, Salmonella y Shiguella. Los análisis de agua se realizaron en las instalaciones de los Laboratorios de la Escuela Superior Politécnica Agropecuaria de Manabí MFL del cantón Bolívar.

Para la realización de estos análisis se utilizaron materiales de muestreo y de laboratorio como botellas de 500 cc, las cuales están provistas de una tapa de apertura remota a fin de no exponer al ambiente su interior una vez tomadas las muestras, para de esta forma, obtener mejores características naturales de del agua. Adicionalmente se utilizó un termómetro para verificar la temperatura de estas aguas y fundas plásticas para transportar las muestras de sedimentos. Así mismo se utilizaron materiales de laboratorio como pipetas, caja Petri, vasos de precipitación, balanzas, entre otros y los medios de cultivo necesarios como agar, caldo selenito y otros.

\section{Resultados y Discusión}

Se muestran los resultados de los análisis de $\mathrm{pH}$ en la playa de la parroquia Los Esteros en los tres puntos de muestreo en los meses de octubre, noviembre y diciembre del año 2014 que es donde se produce el cambio de temporada entre verano invierno. El pH se encuentra en rangos de 6 y 8 que son considerados altos.

En la Figura 3, se muestran los resultados de los análisis de DBO5 en la playa de "Los Esteros" en los meses de octubre, noviembre y diciembre. Los límites máximos permisibles son hasta $100 \mathrm{mg} / \mathrm{lt}$ y en el punto de Muestreo 1 sobrepasa este límite en todos los meses de estudio, mientras que en los meses restantes el resultado cumple con la norma.

A continuación se presentan los resultados de los análisis de DQO en la playa de "Los Esteros" en los meses que se realizó el muestreo. Se resalta que los datos de resultados que presentamos a continuación se hacen en $\mathrm{mg} / \mathrm{lt}$, y el límite máximo permisible que es de $250 \mathrm{mg} / \mathrm{lt}$ según la ley para la Prevención y Control de la Contaminación Ambiental del Ecuador 2008, sobre los índices de calidad del agua en la (Ministerio del Ambiente del Ecuador (MAE), 2008), libro VI. En la Figura 4, se muestran los resultados de sólidos totales encontrados en los diferentes puntos de muestreo de la playa en estudio. Sabiendo que en este parámetro es indeseable que existan sólidos en un cuerpo de agua marino con fines recreativos.

Se aprecia la existencia de una elevada presencia de sólidos totales, siendo el límite máximo permisible de 100 ppm/lt y en los tres meses de muestreo

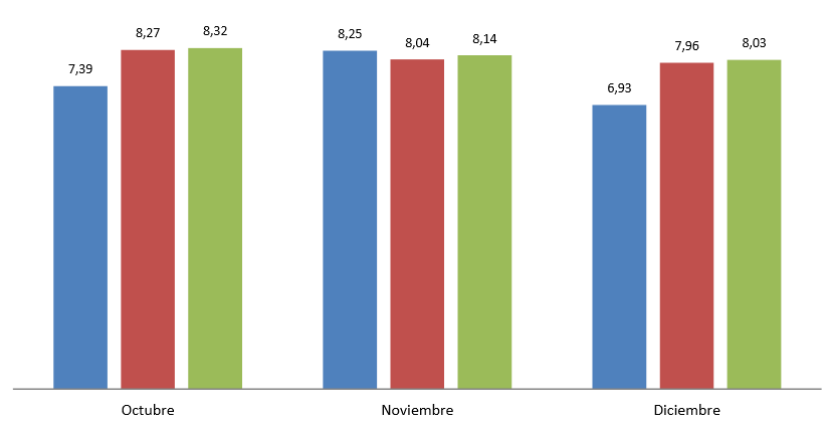

Figura 2. Resultados de análisis de $\mathrm{pH}$. El color azul representa el punto de Muestreo 1 ubicado en la desembocadura del río Muerto, que es el lugar donde se une con la playa de "Los Esteros", el color rojo el punto de Muestreo 2, que está ubicado en la zona de mayor afluencia turística, y el color verde.

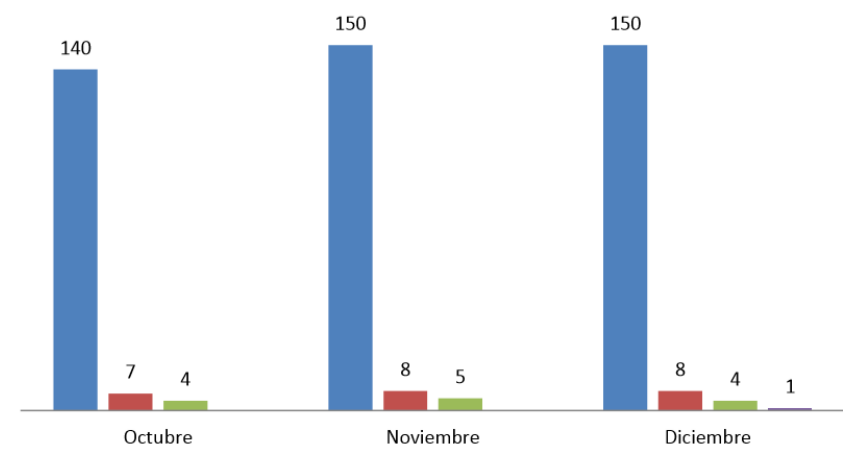

Figura 3. Análisis de $\mathrm{DBO}_{5}$ en $\mathrm{mg} / \mathrm{lt}$. El color azul representa el punto de Muestreo 1 ubicado en la desembocadura del río Muerto, que es el lugar donde se une con la playa de "Los Esteros", el color rojo el punto de Muestreo 2, que está ubicado en la zona de mayor afluencia turística, y el color verde el punto de Muestreo 3 ubicada al final de la playa de dicha parroquia y donde inicia la playa de la parroquia Tarqui. 


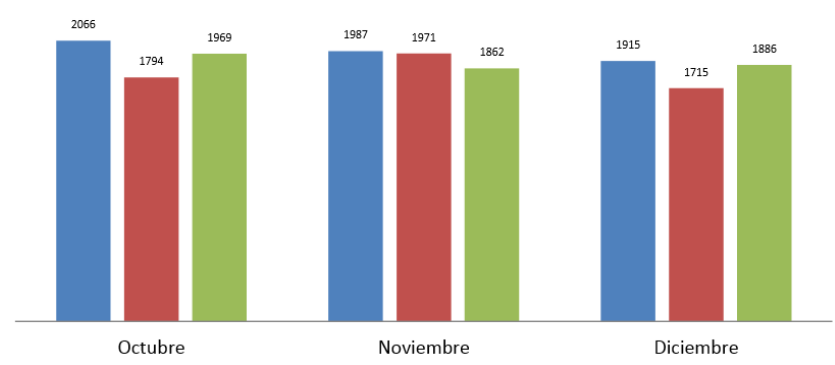

Figura 4. Resultados del análisis de DQO en ppm/lt. El color azul representa el punto de Muestreo 1 ubicado en la desembocadura del río Muerto, que es el lugar donde se une con la playa de "Los Esteros", el color rojo el punto de Muestreo 2, que está ubicado en la zona de mayor afluencia turística, y el color verde el punto de Muestreo 3 ubicada al final de la playa de dicha parroquia y donde inicia la playa de la parroquia Tarqui.

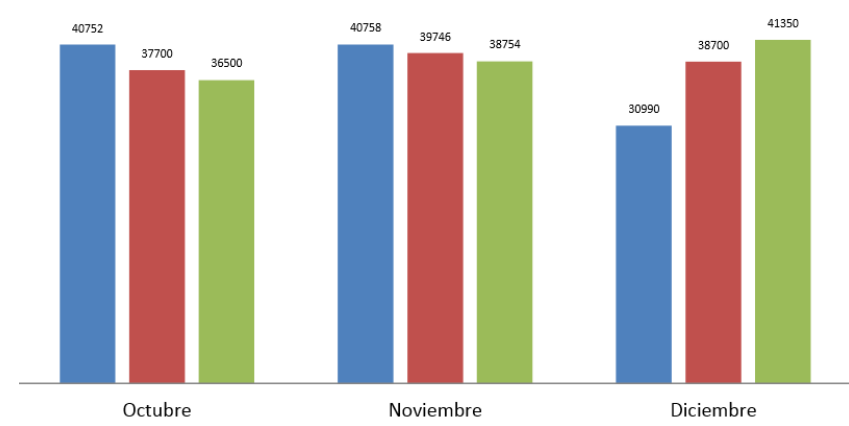

Figura 5. Resultados de análisis de sólidos suspendidos en ppm/lt. El color azul representa el punto de Muestreo 1 ubicado en la desembocadura del río Muerto, que es el lugar donde se une con la playa de "Los Esteros", el color rojo el punto de Muestreo 2, que está ubicado en la zona de mayor afluencia turística, y el color verde el punto de Muestreo 3 ubicada al final de la playa de dicha parroquia y donde inicia la playa de la parroquia Tarqui.

se registraron niveles de entre 1715 y 2055 ppm/lt, asimismo ocurre con los valores de sólidos suspendidos (Figura 5) lo cual demuestra que estas aguas están altamente contaminadas y sobrepasan los parámetros de los índices de calidad.

Así como se realizaron análisis químicos, también se presentan análisis microbiológicos para determinar la presencia de agentes causantes de enfermedades a los habitantes de sectores aledaños, tanto a la playa como al río Muerto por la relación directa y contaminante que se genera en ellos. En el Figura a continuación, se presentan los resultados de los análisis de coliformes fecales, donde se determina la presencia en exceso, y he aquí la respuesta confirmativa a tantos casos registrados en el área de salud en el año anterior a la presente investigación.

En cuanto a Salmonella y Shiguella se tuvieron resultados positivos en el punto de Muestreo 1, es decir en la desembocadura del río hacia la playa ; es decir, que existe presencia de ambos, mientras que en los puntos 2 y 3 no se determinó presencia de los mismos. En cuanto a Coliformes totales en el punto de Muestreo 1, en los tres meses se detectó mediante los análisis de laboratorio que eran incontables pero no por la inexistencia de los mismos sino más bien por la presencia excesiva de los mismos, ya que en el conteo en laboratorio fue imposible determinar un número exacto o aproximado por la elevada cantidad que existe.

En Figura 7 se presentan los datos obtenidos de esta investigación; en el año 2012 según los reportes obtenidos en el subcentro de salud de la parroquia "Los Esteros" que se encuentra ubicado a 500 metros aproximadamente del cauce del río Muerto y a $1 \mathrm{~km}$ aproximado de distancia de la playa en estudio, existieron varias enfermedades reportadas aquel año, y la mayoría de las enfermedades que se relacionan con la contaminación de estos cuerpos marinos se dieron en personas que habitan en el sector del cauce del río Muerto y que muchas veces las viviendas donde ellos habitan, contribuyen a dicha contaminación tanto con los residuos líquidos como residuos sólidos domiciliarios.

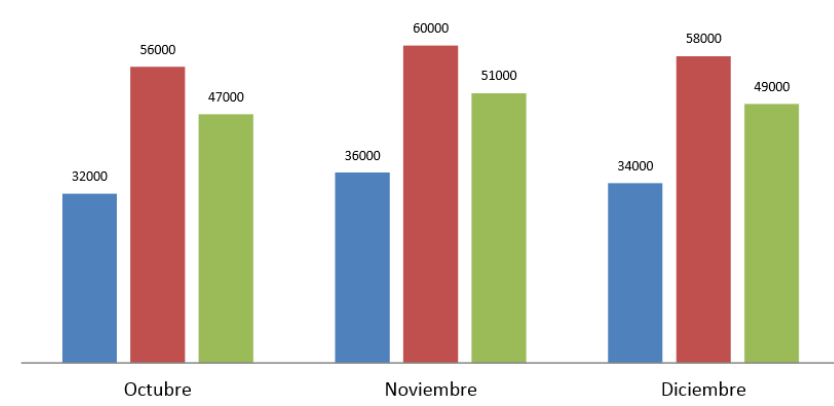

Figura 6. Resultados de análisis de Coliformes fecales. El color azul representa el punto de Muestreo 1 ubicado en la desembocadura del río Muerto, que es el lugar donde se une con la playa de "Los Esteros", el color rojo el punto de Muestreo 2, que está ubicado en la zona de mayor afluencia turística, y el color verde el punto de Muestreo 3 ubicada al final de la playa de dicha parroquia y donde inicia la playa de la parroquia Tarqui. 


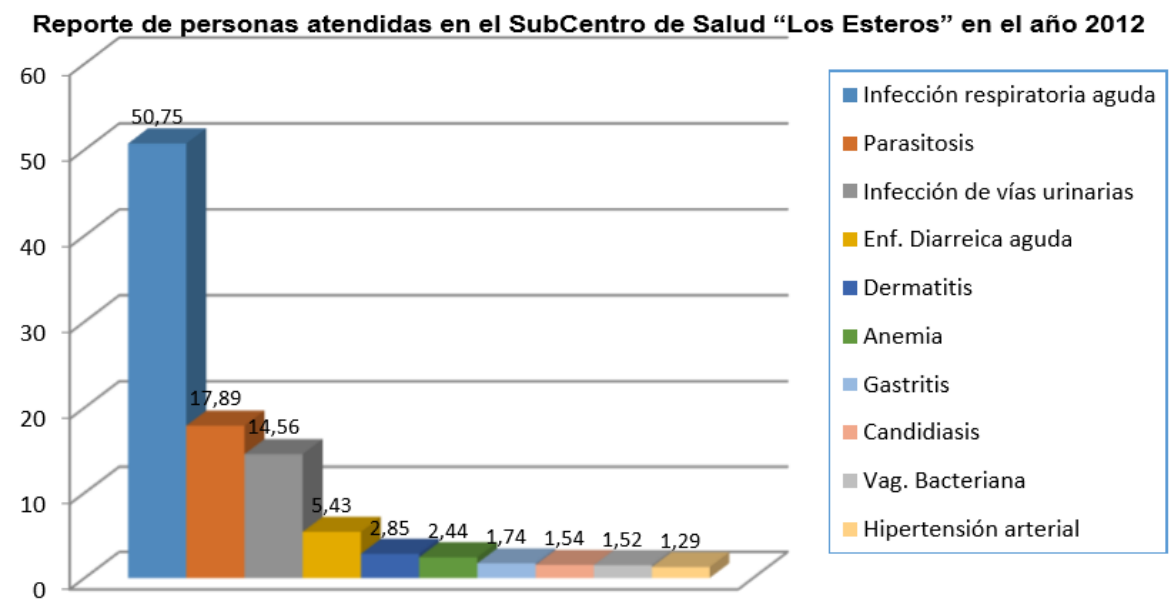

Figura 7. Principales causas de Morbilidad durante el año 2012 SCS Los Esteros-Manta.

En cuanto a enfermedades del tipo Infección respiratoria aguda se presentó aquel año un total de 2244 casos de personas infectadas de la parroquia "Los Esteros" y el mayor índice de casos ocurrió en niños de entre 1-4 años de edad, mientras que parasitosis se dio en su mayoría en niños de 5-14 años de edad donde se tuvieron 395 casos solo en este rango de edad, mientras que de forma general se tuvieron 791 casos. En cuanto a la dermatitis se presentaron 126 casos y de estos la mayor parte fue entre niños de menos de un año hasta personas de entre 15 a 49 años, donde se presentaron 121 casos.

\section{Conclusiones}

Los resultados demuestran que la playa está contaminada tanto en sus características químicas como microbiológicas. Esta playa no debería ser considerada apta para fines recreativos, ya que sobrepasa los límites máximos de contaminación permisibles en la legislación ambiental ecuatoriana, sobretodo en el cauce del Río Muerto. Se concluye que esta playa necesita una intervención inmediata con el fin de concretar su recuperación. Se deben eliminar todos los aspectos y agentes que ocasionan contaminación tanto de origen industrial como por actividades propias de la vivienda.

\section{Referencias}

Centro de Salud Los Esteros. 2013. Archivos y estadísticas. Area de salud. \# 4.
Comisión Nacional del Agua. 1999. Índice de Calidad del Agua. Gerencia de Saneamiento y Calidad del Agua. Recuadro iii: 2.2.2.

Daiefa, Z., A. Borjab, L. Joulamic, M. Azzia, A. Fahdea y H. Bazairid. November 2014. Assessing benthic ecological status of urban sandy beaches (Northeast Atlantic, Morocco) using M-AMBI. Ecological Indicators. 46: 586-595.

Empresa Pública de Aguas de Manta. 05 de 11 de 2014. Lagunas de oxidación de Manta. Manta, Manabí, Ecuador.

González, C.. 2014. Estudio Sobre Causas Y Niveles De Contaminación De La Playa De La Parroquia Los Esteros, Cantón Manta, Periodo JulioDiciembre 2013. Universidad Laica Eloy Alfaro de Manabí. Manta, Ecuador: Proyecto de titulación.

Ley De Gestión Ambiental. 2008. De la gestión ambiental local y nacional. Artículos. 30, 37, 38,40, 43.

L.P.P.C.C.A.E.. 2008. Límites de Descarga a un cuerpo de Agua Marina. Ecuador.

Ministerio del Ambiente del Ecuador (MAE). 2008. Téxto unificado de legislación secundaria TULSMA. 4.

Ramos X. (Entrevistador). 08 de septiembre de 2007. Contaminadas playas Los Esteros y Tarqui. Diario El Universo.

Ruíz, F.. 05 de 11 de 2014. Las aguas residuales de Manta. Entrevista. 